\title{
Gemeinwohlorientierung als Business Case - Corporate Citizenship und dessen Potenziale für Kreditgenossenschaften
}

Bürgerschaftliches Engagement von Unternehmen; Corporate Citizenship; Genossenschaften und Gemeinwohlorientierung; gesellschaftliche Verantwortung von Unternehmen; Kreditgenossenschaften

Corporate Citizenship ist in den letzten Jahren verstärkt in den Fokus öffentlicher, wirtschaftlicher und wissenschaftlicher Debatten gerückt. In Abgrenzung zu sonstigen Formen gesellschaftlichen Engagements steht hierbei v.a. die Verbindung von unternehmerischem mit gesellschaftlichem Nutzen im Vordergrund. Der vorliegende Beitrag geht zunächst auf zwei wichtige theoretische Ansätze zur Erklärung dieser Synthese ein: den Stakeholder Management-Ansatz sowie Ansätze, die unter dem Begriff des Sozialen Kapitals subsumiert werden. Dem folgt eine Übertragung auf Kreditgenossenschaften, wobei der Schwerpunkt auf der Frage liegt, welche Potenziale durch Corporate Citizenship für Kreditgenossenschaften bestehen. Es wird aufgezeigt, wie eine gemeinwirtschaftliche Zielorientierung derselben durch Corporate Citizenship mit mitglieder- und unternehmensorientierten Zielen verbunden werden kann.

\section{Einleitung}

Die anhaltende Leistungskrise des deutschen Wohlfahrtsstaates macht es erforderlich, dass Aufgaben, die von diesem traditionell übernommen wurden, zumindest teilweise auf andere gesellschaftliche Akteure übertragen werden. In diesem Zusammenhang ist das Konzept der Bürgergesellschaft verstärkt in den Fokus öffentlicher Debatten gerückt. So hat bspw. der Deutsche Bundestag, neben zahlreichen weiteren Initiativen, eine EnqueteKommission „Zukunft des Bürgerschaftlichen Engagements“ eingesetzt. ${ }^{1}$ Im Kontext einer solchen Rollenverschiebung gesellschaftlicher Akteure wird insbesondere auch die Rolle privatwirtschaftlicher Unternehmen in der Gesellschaft, d.h. als „Corporate Citizens“ diskutiert. Um Unternehmen langfristig und nachhaltig zur Übernahme von Aufgaben des Gemeinwohls motivieren zu können, ist es nicht nur für Unternehmen, sondern auch für das Allgemeinwohl von Interesse, unternehmerisches Bürgerengagement so auszurichten, dass hierdurch gesellschaftlicher mit unternehmerischem Nutzen verbunden werden kann. An dieser Synthese setzt das Konzept des Corporate Citizenship an. Ansatz ist dabei nicht, Gemeinwohlorientierung für ökonomische Zwecke zu instrumentalisieren,

1 Vgl. hierzu Enquete-Kommission „Zukunft des Bürgerschaftlichen Engagements“ Deutscher Bundestag (2002). 
sondern es wird von einer positiven Relation zwischen gemeinwirtschaftlichen und ökonomischen Zielen ausgegangen: Durch die Übernahme gemeinwirtschaftlicher Aufgaben investieren Unternehmen in ihr gesellschaftliches Umfeld mit dem Ziel der langfristigen Schaffung, Verbesserung oder Stabilisierung von gesellschaftlichen Rahmenbedingungen ihrer eigenen wirtschaftlichen Tätigkeit. ${ }^{2}$ Gemeinwohlorientierung von Unternehmen wird somit zum möglichen Business Case erhoben. Diese Synthese zwischen gemeinwohlorientierten und sonstigen Unternehmenszielen steht, konkret für die Gruppe der Kreditgenossenschaften, im Mittelpunkt dieses Beitrags.

\section{Begriffsbestimmung}

Während in den USA entsprechende Debatten bereits Ende der 70er Jahre des letzten Jahrhunderts geführt wurden, hielt der Begriff des Corporate Citizenship in Deutschland erst in den 90er Jahren Einzug in die wissenschaftliche und öffentliche Diskussion. ${ }^{3}$ Er hat bisher weder international noch im deutschsprachigen Raum einheitliche Verwendung gefunden. In Deutschland wird - im Gegensatz zur internationalen Debatte - meist von einem engeren Begriffsverständnis ausgegangen, welches Corporate Citizenship an der Schnittstelle von Unternehmen zu anderen gesellschaftlichen Akteuren verortet und hierunter bürgerschaftliches Engagement von Unternehmen fasst, welches auf das Lösen konkreter Probleme des Gemeinwesens ausgerichtet ist. ${ }^{4}$

Corporate Citizenship grenzt sich dabei zu herkömmlichen Formen gesellschaftlichen Engagements v.a. durch drei wesentliche Merkmale ab: Erstens wird, wie bereits dargestellt, postuliert, dass gesellschaftliches Engagement im Rahmen von Corporate Citizenship so gestaltet wird, dass es nicht nur auf gesellschaftlichen sondern auch auf unternehmerischen Nutzen ausgerichtet ist. Es wird in diesem Zusammenhang auch von „WinWin"-Situationen gesprochen. ${ }^{5}$ Die damit verbundene Überholung traditionellen Denkens in Nullsummenspielen hinsichtlich gesellschaftlichen Engagements von Unternehmen liegt nicht nur im Interesse der beteiligten Wirtschaft: Das Erreichen wechselseitigen Vorteils wird gleichzeitig als Garant dafür gesehen, dass Unternehmen sich langfristig für Belange ihres gesellschaftlichen Umfeldes einsetzen, dies auch bzw. gerade in wirtschaftlichen Krisenzeiten. ${ }^{6}$ Zweites Merkmal, das aus dem ersten Merkmal folgt, ist die strategische Ausrichtung von Corporate Citizenship. Unter der Prämisse einer effektiven und effizienten Gestaltung geht es nicht um isolierte Maßnahmen gesellschaftlichen Engagements, sondern um deren Planung und Bündelung im Rahmen einer einheitlichen Strategie. Drittes Merkmal ist die Freiwilligkeit der Maßnahmen. Diese bezieht sich sowohl auf

2 Vgl. auch Habisch (2003), S. 54 ff.

3 Vgl. hierzu Backhaus-Maul (2004).

4 Vgl. exemplarisch Westebbe/Logan (1995), S. 17. Ein weiter gefasstes Begriffsverständnis vertritt in der deutschsprachigen Debatte insbesondere P. Ulrich (vgl. Ulrich (2000)).

5 Vgl. exemplarisch Habisch (2003), S. 54 ff.

6 Vgl. Fabisch (2004), S. 36. So unterhält bspw. das Bundesministerium für Wirtschaft und Technologie (BMWi) im Rahmen seiner Mittelstandsoffensive ,pro mittelstand“ eine öffentlich zugängliche Corporate Citizenship-Datenbank mit Praxisbeispielen aus der freien Wirtschaft, um bürgerschaftliches Engagement von Unternehmen zu fördern (vgl. BMWi (21. Januar 2006)). 
gesetzliche wie auf marktliche Regelungen. Corporate Citizenship geht also über gesetzliche Regelungen hinaus und unterliegt gleichzeitig keinen marktlichen Mechanismen.

Corporate Citizenship umfasst nach diesem enger gefassten Begriffsverständnis unternehmerische Aktivitäten, die durch folgende Merkmale gekennzeichnet werden:

- Ausrichtung auf die Lösung gesellschaftlicher Probleme sowie die Mitgestaltung gesellschaftlicher Prozesse

- Anstreben wechselseitigen Vorteils für wirtschaftliche, zivilgesellschaftliche sowie öffentliche Akteure, d.h. sog. „Win-Win-Situationen“

- Freiwilligkeit

- $\quad$ strategische Ausrichtung. ${ }^{7}$

\section{Erklärungsansätze}

Die genannte Synthese zwischen unternehmerischem und gesellschaftlichem Nutzen im Konzept des Corporate Citizenship lässt sich unter Einbeziehung verschiedener Theorieansätze erklären. Meist werden hierzu der Stakeholder Management-Ansatz sowie Ansätze Sozialen Kapitals herangezogen.

\section{a) Stakeholder Management-Ansatz}

Eng verbunden mit den Konzepten des Corporate Citizenship und der damit in Zusammenhang stehenden Frage, in welchem Umfang und in welcher Weise unternehmerisches Handeln mit gesellschaftlichen Entwicklungen verbunden ist, ist der Stakeholder Management-Ansatz. ${ }^{8}$ Dieser wurde insbesondere durch Freeman geprägt und hat seitdem verstärkt Aufmerksamkeit erhalten. ${ }^{9}$ Ausgangspunkt der Überlegungen ist hierbei, dass für Unternehmen nicht nur die traditionellen Anspruchsgruppen wie Kapitalgeber, Lieferanten, Mitarbeiter und Kunden von Bedeutung sind, sondern dass darüber hinaus auch andere Anspruchs- bzw. Stakeholdergruppen Einflüsse auf Unternehmen haben können. Hierzu gehören insbesondere auch Stakeholdergruppen wie zivilgesellschaftliche Vereine, die örtliche Gemeinschaft oder die Öffentlichkeit. Deren Interessen und Ansprüche an das Unternehmen sind daher in unternehmerische Planungsprozesse einzubeziehen, um die Beziehung zwischen den verschiedenen Anspruchsgruppen und dem Unternehmen sowie deren gegenseitigen Einfluss möglichst positiv zu gestalten. Hierzu können folglich auch Interessen im Hinblick auf das Gemeinwesen, wie sie im Zentrum des Corporate Citizenship-Konzeptes stehen, gehören. Dementsprechend beschreibt bspw. Carroll

7 Vgl. hierzu, insbesondere zu den Aspekten des wechselseitigen Nutzens und der strategischen Integration, exemplarisch Westebbe/Logan (1995); Maaß/Clemens (2002); Backhaus-Maul (2004); Habisch (2003) sowie Wieland (2002).

8 Näsi bezeichnet aus diesem Grund den Stakeholder Management-Ansatz als das „,vereinende Prinzip“ für den Bereich Wirtschaft und Gesellschaft und führt die Herstellung dieser Verbindung auf Carroll zurück (vgl. Näsi (1995), S. 21). Ähnlicher Ansicht ist auch Freeman (2004).

9 Vgl. Freeman (1984). Für einen Überblick über die Diskussion des Stakeholder Management-Ansatzes im deutschsprachigen Raum s. Hansen/Bode/Moosmayer (2004). 
Corporate Citizenship als Konzept, welches die Beziehungen des Unternehmens zu all seinen wichtigen Stakeholdern adressiert. ${ }^{10}$

\section{b) Ansätze Sozialen Kapitals}

Obwohl der Begriff des Sozialen Kapitals bereits in den 60er Jahren Einzug in die wissenschaftliche Debatte gehalten hat, liegt hierfür bis heute weder in der Forschung noch in der Praxis ein einheitliches Begriffsverständnis vor, so dass unter diesem Begriff verschiedene Konzepte subsumiert werden. ${ }^{11}$ Allgemein wird unter Sozialem Kapital die Befähigung und Bereitschaft zur Kooperation verstanden, welche aus der Zugehörigkeit von Akteuren zu sozialen Netzwerken resultieren. Grundidee ist dabei, dass soziale Netzwerke im zivilgesellschaftlichen und familiären Bereich individuelle und kollektive Vorteile innerhalb einer Gemeinschaft mit sich bringen, die sich auch auf den wirtschaftlichen und staatlichen Sektor erstrecken können. Denn in der heutigen modernen Gesellschaft, in der traditionelle Strukturen aufgebrochen werden und pluralistische Wertvorstellungen herrschen, ist es nicht mehr möglich sich auf unvergängliche Werte zu verlassen, die das Verhalten von Akteuren kontrollieren. ${ }^{12}$ Somit ergibt sich das Problem der Verhaltensregelung von Akteuren in einer deregulierten Umwelt. ${ }^{13}$ Bedingungen kollektiver Handlungen müssen neu zwischen den Akteuren bestimmt werden. ${ }^{14}$ Hierzu tragen nicht nur staatliche Regelungen bei, sondern auch informelle Selbstbindungsmechanismen. ${ }^{15}$ Als „Good Corporate Citizen“ richtet ein Unternehmen das eigene Handeln daher nicht nur an formalen Regeln und Normen aus, sondern auch an ,selbst geschaffenen" Regeln, mit denen es Erwartungen und Ansprüche seines sozio-ökonomischen Umfeldes aufgreift. ${ }^{16}$ Die Entwicklung sozialer Ordnungsformen aus der Bürgerschaft heraus führt dabei nicht zu einer Ablösung staatlicher Ordnung, sondern steht in einem Komplementärverhältnis zu dieser. ${ }^{17}$ Staatliche Rahmenbedingungen können den Aufbau selbstregulierender Netzwerke im Sinne Sozialen Kapitals fördern, welche wiederum die Funktionen staatlicher Ordnung stabilisieren, so dass hier ein Rückkopplungseffekt besteht. ${ }^{18}$ Zur Förderung bürgerschaftlichen Engagements von Unternehmen ist somit ein Staat hilfreich, der in diesem Prozess eine aktive Rolle spielt und förderliche Rahmenbedingungen schafft. ${ }^{19}$ Gleichzeitig können sich Effekte der Überregulierung jedoch auch negativ auswirken. ${ }^{20}$

10 Vgl. Carroll (1998), S. 1.

11 Für einen Überblick über Positionen wichtiger Vertreter der Debatte um Soziales Kapital vgl. die Übersichtsarbeit von Haug (1997). Zu den Hauptvertretern dieser Forschungsrichtung zählen J. Coleman, R. D. Putnam sowie P. Bourdieu.

12 Vgl. Habisch/Schmidpeter (2001), S. 14.

13 Vgl. Englert (2001), S. 35.

14 Vgl. Habisch/Schmidpeter (2001), S. 14.

15 Vgl. Habisch (2003), S. 23 und S. 37.

16 Ringlstetter/Schuster (2003), S. 193.

17 Vgl. Habisch (2003), S. 37 sowie ders. (1998), S. 123.

18 Vgl. Schmidpeter (2001), S. 124.

19 Vgl. Lang/Kromminga (2003), S. 93.

20 Vgl. Habisch (1998), S. 114. 
Corporate Citizenship sowie die so aufgebauten sozialen Beziehungen und die damit verbundenen Normen, Werte und gegenseitigen Verhaltenserwartungen können somit verstanden werden als Investition in Soziales Kapital. ${ }^{21}$ Von einer in diesem Sinne erfolgreichen Investition profitieren Gesellschaft, Staat sowie das Unternehmen, denn indem das Soziale Kapital der Gesellschaft gesteigert wird, wird gleichzeitig die nichtwirtschaftliche Basis der Marktwirtschaft gestärkt. ${ }^{22}$ Weiterer Nutzen für Unternehmen kann im Zugang zu qualifiziertem Personal oder der Etablierung langfristiger Beziehungen zu Netzwerkpartnern liegen. ${ }^{23}$ Wird Corporate Citizenship als eine solche Investition in Soziales Kapital verstanden, so stellt es keinen ,altruistic luxury of good citizens“ dar, sondern trägt zur nachhaltigen Steigerung des Shareholder Value bei. ${ }^{24}$

\section{Potenziale für Kreditgenossenschaften}

Im kreditgenossenschaftlichen Bereich hat bürgerschaftliches Engagement einen hohen Stellenwert und wird in der Praxis intensiv angegangen, sowohl von den einzelnen Kreditgenossenschaften als auch über Initiativen, die von den kreditgenossenschaftlichen Verbänden ausgehen oder im Verbund gestaltet werden, so bspw. durch die Gründung des Vereins Aktive Bürgerschaft, der sich als Kompetenzzentrum für Corporate Citizenship und bürgerschaftliches Engagement innerhalb der genossenschaftlichen Bankengruppe versteht. ${ }^{25}$

De jure ist das Primärziel von Kreditgenossenschaften durch $§ 1$ GenG vorgegeben als die Förderung des Erwerbs und der Wirtschaft ihrer Mitglieder. Eine solche geschieht in der Regel vorrangig durch die Leistungserbringung im Rahmen eines bankenwirtschaftlichen Produkt- bzw. Dienstleistungsportfolios sowie daneben durch die Auszahlung einer Dividende an die Mitglieder. Die Sicherung einer solchen Leistungserbringung ist für Kreditgenossenschaften aufgrund gesetzlicher und wirtschaftlicher Rahmenbedingungen allerdings häufig mit der Verfolgung weiterer Ziele wie insbesondere Gewinn- bzw. Rentabilitätszielen, aber auch Markt- und Leistungszielen verbunden. ${ }^{26}$ Dementsprechend sollen im Folgenden Potenziale des Corporate Citizenship im Hinblick auf mitgliederorientierte wie auch auf unternehmensorientierte Ziele von Kreditgenossenschaften betrachtet werden.

21 Vgl. Habisch/Schmidpeter (2001), S. 17 sowie Habisch (1998).

22 Vgl. Renner (2001), S. 65.

23 Vgl. Pinkepank (2001), S. $116 \mathrm{f}$.

24 Ebd. S. 115; vgl. hierzu auch Habisch (1998), S. 125.

25 Vgl. hierzu Aktive Bürgerschaft (2003).

26 Vgl. z.B. Leupold (1975), S. 52 sowie Dülfer (1980), Sp. 1864. 
a) Nutzenpotenziale im Hinblick auf mitgliederorientierte Ziele

Hinsichtlich mitgliederorientierter Ziele können Potenziale von Corporate Citizenship sowohl ökonomischer als auch außerökonomischer Art sein. Da Geschäftssitz der Kreditgenossenschaft und Lebensmittelpunkt bzw. Tätigkeitsschwerpunkt der Mitglieder in der Regel identisch sind, kann v.a. regional ausgerichtetes Corporate Citizenship direkt den Mitgliedern zugute kommen. So kann eine Verbesserung der gesellschaftlichen Rahmenbedingungen der wirtschaftlichen Tätigkeit der Mitglieder erreicht werden, wenn z.B. die Unterstützung regionaler Infrastrukturmaßnahmen durch die Kreditgenossenschaft zur wirtschaftlichen Förderung der Region und damit zum Erhalt von Arbeitsplätzen der Mitglieder beiträgt. ${ }^{27}$

Weiter können Potenziale von Corporate Citizenship für Kreditgenossenschaften auch darin liegen, dass die Interessen der Mitglieder in ihrer Umwelt, auch über wirtschaftliche Bedürfnisse hinausgehend, berücksichtigt werden. ${ }^{28}$ So kann Corporate Citizenship bspw. zur Erfüllung von Mitgliederbedürfnissen beitragen, wenn hierdurch konkrete Probleme des Gemeinwesens, die auch die Mitglieder betreffen, gelöst werden können. So kann z.B. die Lebensqualität der Mitglieder durch die Förderung regionaler Freizeitangebote im Rahmen von Corporate Citizenship erhöht werden. Darüber hinaus muss die Motivation von Mitgliedern zur Beteiligung an der Kreditgenossenschaft nicht unbedingt ökonomisch-rational begründet sein, sondern können hiermit auch darüber hinausgehende sozio-ökonomische Motivationen verbunden $\operatorname{sein}^{29}$ so dass eine Gemeinwohlorientierung der Kreditgenossenschaft sehr wohl im Interesse der Mitglieder liegen kann, auch ohne dass diese auf deren wirtschaftliche Förderung ausgerichtet ist.

Zusammengefasst liegen Potenziale des Corporate Citizenship im Hinblick auf mitgliederorientierte Ziele somit in der Förderung bzw. Verbesserung der Rahmenbedingungen der Wirtschaft der Mitglieder sowie in der Berücksichtigung außerökonomischer Interessen und der Erfüllung dementsprechender Bedürfnisse der Mitglieder.

b) Nutzenpotenziale im Hinblick auf unternehmensorientierte Ziele

Nutzenpotenziale hinsichtlich unternehmensorientierter Ziele von Kreditgenossenschaften können grundsätzlich unterschieden werden in Potenziale hinsichtlich einzelbetrieblicher Ziele und solche hinsichtlich gesamtwirtschaftlicher Ziele. ${ }^{30}$

27 In diesem Sinne bezieht bspw. Dülfer gesellschaftsorientierte, ökononomische Ziele in den Förderauftrag ein, wenn er hierunter auch Ziele, die sich ,,auf die Stellung des Mitglieds ... in seiner wirtschaftlichen Umwelt (z.B. Existenzsicherung, Beeinflussung der Wirtschaftsordnung, Verbesserung der Marktsituation) beziehen“, fasst (vgl. ebd., Sp. 1863).

28 So sieht Richter die „Wahrung sowie Verbesserung der nicht nur materiellen Bedürfnisse der Mitglieder“ als Ziele im Rahmen des Förderauftrags, da dieser auf die Erhaltung bzw. Erhöhung der Lebensqualität der Mitglieder ausgerichtet sei (vgl. Richter (1981), S. 157 ff.). Er macht in diesem Zusammenhang auch darauf aufmerksam, dass ökonomische Zwecke nicht um ihrer selbst Willen verfolgt werden, so dass hiermit im Allgemeinen letztlich wieder eine außerökonomische Zielsetzung verbunden ist.

30 Vgl. Schrader (2003), S. 78 ff. sowie Habisch (2003), S. 61 ff. 
Potenziale im Hinblick auf gesamtwirtschaftliche Ziele resultieren dabei vorwiegend aus dem Aufbau Sozialen Kapitals. Durch langfristige Selbstbindungsmechanismen und die Zusammenarbeit mit zivilgesellschaftlichen Akteuren stärken Kreditgenossenschaften die gesellschaftliche Basis der Marktwirtschaft und verbessern damit die Rahmenbedingungen ihres eigenen wirtschaftlichen Handelns. Dieses Nutzenpotenzial zielt somit auf die institutionellen Interessen der Kreditgenossenschaft. Die Lösung von Problemen des Gemeinwesens innerhalb ihres Geschäftsgebiets, seien diese wirtschaftlicher oder sozialer Art, ist für Kreditgenossenschaften dabei von vitaler Bedeutung, da sie sich - ähnlich wie Sparkassen - aufgrund ihrer regionalen Verankerung nicht wie andere Kreditinstitute aus bestimmten Geschäftsgebieten zurückziehen können. Sie besitzen somit ein ,genuines Interesse an der Wohlfahrt der sie unmittelbar umgebenden Gesellschaft, da aus dieser ihre Mitglieder und Kunden stammen“. ${ }^{31}$

Das Soziale Kapital einer Region ist aber auch aus anderen Gründen ein wichtiger Faktor für den Erfolg von Kreditgenossenschaften. Universalkreditgenossenschaften sind durch eine starke Heterogenität in der Mitgliedschaft gekennzeichnet. Subjektive Strukturmerkmale sind hier neben dem Fehlen der gegenseitigen Kenntnis der Mitglieder (Anonymität) die unterschiedliche wirtschaftliche und gesellschaftliche Position derselben sowie daraus resultierende heterogene Interessenlagen, weiterhin das Fehlen eines gemeinsamen normierten Verhaltens, der Verlust der Mitgliederbindung und Identifikation mit der Genossenschaft, woraus die Veränderung der Mitgliedsverhältnisses zu einer reinen Kundenbeziehung folgt. ${ }^{32}$ Dieser Effekt wird durch Fusionen sowie steigende Anforderungen der Bankenaufsicht verstärkt. ${ }^{33}$ Bonus macht daher - ohne den Begriff des Sozialen Kapitals explizit zu nennen - auf die Bedeutung desselben für lokal verankerte Kreditgenossenschaften aufmerksam, wenn er die örtliche Gemeinschaft, die sich als Dorf-, Kleinstadt- oder Stadtteilgemeinschaft zusammengehörig fühlt, als „wichtige Klammer zwischen Unternehmen und Mitgliedern neben der eigentlichen Förderung“" nennt. ${ }^{34}$ Corporate Citizenship kann somit indirekt zum Aufbau von Vertrauen und geteilten Wertvorstellungen innerhalb der Mitgliedergruppe und damit zu einer stärkeren Mitgliederbindung beitragen.

Bezüglich einzelbetrieblicher Ziele bietet Corporate Citizenship v.a. Nutzenpotenziale im Hinblick auf Reputations- und Kommunikationsziele, mitarbeiterorientierte Ziele sowie produkt- und marktorientierte Ziele. Im Hinblick auf kommunikations- und reputationsorientierte Ziele wird Corporate Citizenship allgemein ein sehr hohes Nutzenpotenzial beigemessen. ${ }^{35}$ Dies belegen u.a. empirische Studien von Maaß und Clemens sowie von Fabisch, in denen teilweise über $90 \%$ der Befragten angeben, mit Corporate Citizenship öffentlichkeitsbezogene Ziele, d.h. Imageverbesserung, Dokumentation gesellschaftlicher Verantwortung sowie Verbesserung des Unternehmerbildes, zu

31 Wagner (1999), S. 162.

32 Vgl. Pauli (1980), Sp. 1207.

33 Vgl. Bonus (1994), S. 88.

34 Vgl. ebd., S. 88.

35 Vgl. hierzu Westebbe/Logan (1995), S. 22 ff.; Fabisch (2004), S. 121 ff.; Hansen/Schrader (2005), S. 383. 
verbinden.$^{36}$ Corporate Citizenship schafft hierbei die Möglichkeit, sich positiv vom Wettbewerb abzugrenzen und sich als „guter Unternehmensbürger“ zu positionieren. ${ }^{37}$ Dies wird gerade auch für Kreditgenossenschaften vor dem Hintergrund des Verdrängungswettbewerbs im Banksektor wichtig, da es kaum noch Möglichkeiten gibt, sich ausschließlich über Bankprodukte vom Wettbewerb abzugrenzen. Corporate Citizenship kann hierbei eine Möglichkeit bieten, genossenschaftliches Gedankengut, wie z.B. die Leitbilder der Solidarität und Selbsthilfe, mehr in den Vordergrund zu stellen und Kreditgenossenschaften somit wieder ein mehr genossenschaftliches Profil zu geben.

Im Hinblick auf mitarbeiterorientierte Ziele kann Corporate Citizenship unterschiedliche Nutzenpotenziale aufweisen. Insbesondere mit dem Instrument des Corporate Volunteering, d.h. der Unterstützung und Förderung bürgerschaftlichen Engagements der Mitarbeiter eines Unternehmens durch dasselbe bzw. deren Einbindung in Corporate Citizenship-Aktivitäten der Kreditgenossenschaft, ${ }^{38}$ werden positive Effekte im Hinblick auf die Entwicklung sozialer Kompetenzen von Mitarbeitern und Führungskräften verbunden. ${ }^{39}$ Denn durch die Ausübung gesellschaftlichen Engagements erlernen sie Kompetenzen, die auch für ihren Arbeitsalltag von Bedeutung sind. Gleichzeitig werden Team- und Kommunikationsfähigkeit geschult, da bürgerschaftliches Engagement in der Regel nicht isoliert, sondern in Zusammenarbeit mit anderen stattfindet. Mitarbeiter werden so insgesamt im Umgang mit Menschen trainiert, was vor dem Hintergrund beratungsintensiver Finanzprodukte und der starken Mitglieder- und Kundenorientierung von Kreditgenossenschaften von besonderer Bedeutung ist.

Weiter kann Corporate Citizenship zur Erhöhung der Mitarbeitermotivation beitragen. Hierfür sind v.a. zwei unterschiedliche Effekte verantwortlich: So können Corporate Citizenship-Aktivitäten der Kreditgenossenschaft sich positiv darauf auswirken, dass die Mitarbeiter ihren Arbeitgeber als gesellschaftlich verantwortliches Unternehmen erleben, mit dem sie sich identifizieren können und möchten. ${ }^{40}$ Daneben stammen die Mitarbeiter von Kreditgenossenschaften oftmals aus dem lokalen Umfeld derselben und sind somit häufig selbst über ihre Einbindung in die örtliche Gemeinschaft Leistungsempfänger des bürgerschaftlichen Engagements. Der Aufbau gemeinsamer Wertvorstellungen hat so auch positive Effekte auf die Unternehmenskultur. ${ }^{41}$ Dieses Nutzenpotenzial ist für die genossenschaftliche Bankengruppe aufgrund der in den letzten Jahren stattgefundenen zahlreichen Fusionen besonders wichtig. ${ }^{42}$

Im Hinblick auf produkt- und marktorientierte Ziele kann Corporate Citizenship durch eine Erhöhung der Reputation der Kreditgenossenschaft sowie über die Identifikationspotenziale, die gesellschaftliches Engagement für Kunden und Mitglieder bietet, zur Verbesserung bestehender Mitglieder- und Kundenbeziehungen und damit zu einer stärkeren

36 Vgl. Maaß/Clemens (2002), S. 81 ff. sowie Fabisch (2004), S. 225 f.

37 Vgl. McIntosh et al. (1998), S. $61 \mathrm{ff}$.

38 Vgl. hierzu Fabisch (2004), S. 92 ff.; Habisch (2003), S. 128 f. sowie Schrader (2003).

39 Vgl. Schrader (2003), S. 89 ff. sowie Habisch (2003), S. 66 ff.

40 Vgl. Schrader (2003), S. $91 \mathrm{ff}$.

41 Vgl. Rösner (1997).

42 Vgl. hierzu auch Habisch (2003), S. 69 f. sowie Blome-Drees/Schmale (2004) zur Unternehmenskultur von Kreditgenossenschaften bei Fusionsprozessen. 
Bindung derselben an die Kreditgenossenschaft beitragen. ${ }^{43}$ Da für Kreditgenossenschaften aufgrund ihrer lokalen Verankerung ein Wechsel des Standorts in der Regel nicht in Frage kommt, sind sie - zusammen mit den Sparkassen - stärker als die Kreditbanken auf das Vertrauen ihrer Kunden angewiesen, da für sie keine Marktalternativen zur Verfügung stehen. Darüber hinaus können so auch neue Kunden gewonnen werden, da Kaufund Investitionsentscheidungen heutzutage immer mehr auch auf Grundlage des ,guten Rufs" von Unternehmen getroffen werden. ${ }^{44}$

Weiter kann Corporate Citizenship einen verbesserten Zugang zu Informationen über wichtige Zielgruppen verschaffen. Denn durch kooperative Formen von Corporate Citizenship, z.B. durch die Zusammenarbeit der Kreditgenossenschaft mit Bürgern und gemeinnützigen lokalen Vereinen in Bürgerstiftungen, kann Zugang zu informellen Informationen über verschiedene Zielgruppen wie lokale Bevölkerungsschichten und -gruppen ermöglicht werden, die auf formalen Wegen nur sehr schwer zu erhalten sind. Dementsprechend können Produkte und Dienstleistungen effektiver und auch effizienter gestaltet werden. ${ }^{45}$ So übernimmt Corporate Citizenship eine wichtige Informationsfunktion für Kreditgenossenschaften, welche vor dem Hintergrund von Informationen als immer wichtiger werdende Ressource von besonderer Bedeutung ist. ${ }^{46}$

Insgesamt lassen sich somit vielfältige Nutzenpotenziale von Corporate Citizenship für Kreditgenossenschaften feststellen, die sich auf mitgliederorientierte wie auch auf unternehmensorientierte Ziele beziehen. Diese sind in Abbildung 1 zusammengefasst.

\section{Fazit}

Die Frage, inwiefern Kreditgenossenschaften als gemeinwirtschaftliche Unternehmen betrachtet werden können oder nicht, ist seit langem Gegenstand der genossenschaftswissenschaftlichen Diskussion und bis heute nicht einheitlich beantwortet. ${ }^{47}$

Die obigen Ausführungen haben gezeigt, dass Kreditgenossenschaften durch Corporate Citizenship nicht nur zum Gemeinwohl beitragen können, sondern dass umgekehrt eine Gemeinwohlorientierung derselben auch zur Förderung der Mitglieder und des Genossenschaftsbetriebs beiträgt. In diesem Sinne stellt sich hinsichtlich Corporate Citizenship weniger die Frage, wie zusätzlich zum Förderauftrag Kreditgenossenschaften zum Gemeinwohl beitragen können oder inwiefern der Förderauftrag als Gemeinwohlorientierung zu interpretieren ist. Sondern unter Effizienzgesichtspunkten ist eine Gemeinwohlorientierung folglich gerade auch für nicht-gemeinwirtschaftliche Zielsetzungen derselben erforderlich, um Potenziale im Hinblick auf mitglieder- und unternehmensorientierte Ziele auszuschöpfen. Danach wäre Gemeinwohlorientierung für Kreditgenossenschaften nicht bloß Nebeneffekt ihrer genossenschaftlichen Tätigkeit, sondern diese würde be-

43 Vgl. Hansen/Schrader (2005), S. 384.

44 Vgl. Maaß/Clemens (2002), S. 85 f.

45 Vgl. Logan/Roy/Regelbrugge (1997), S. 87.

46 Vgl. Habisch (2003), S. $74 \mathrm{f}$.

47 Vgl. hierzu exemplarisch Engelhardt (1983); Thiemeyer (1990); Weisser (1977); Mändle (1992) sowie Schwarz (2005). 
wusst, auch bspw. bei erwerbs- oder förderungswirtschaftlicher Orientierung ${ }^{48}$, im Zielsystem derselben verankert.

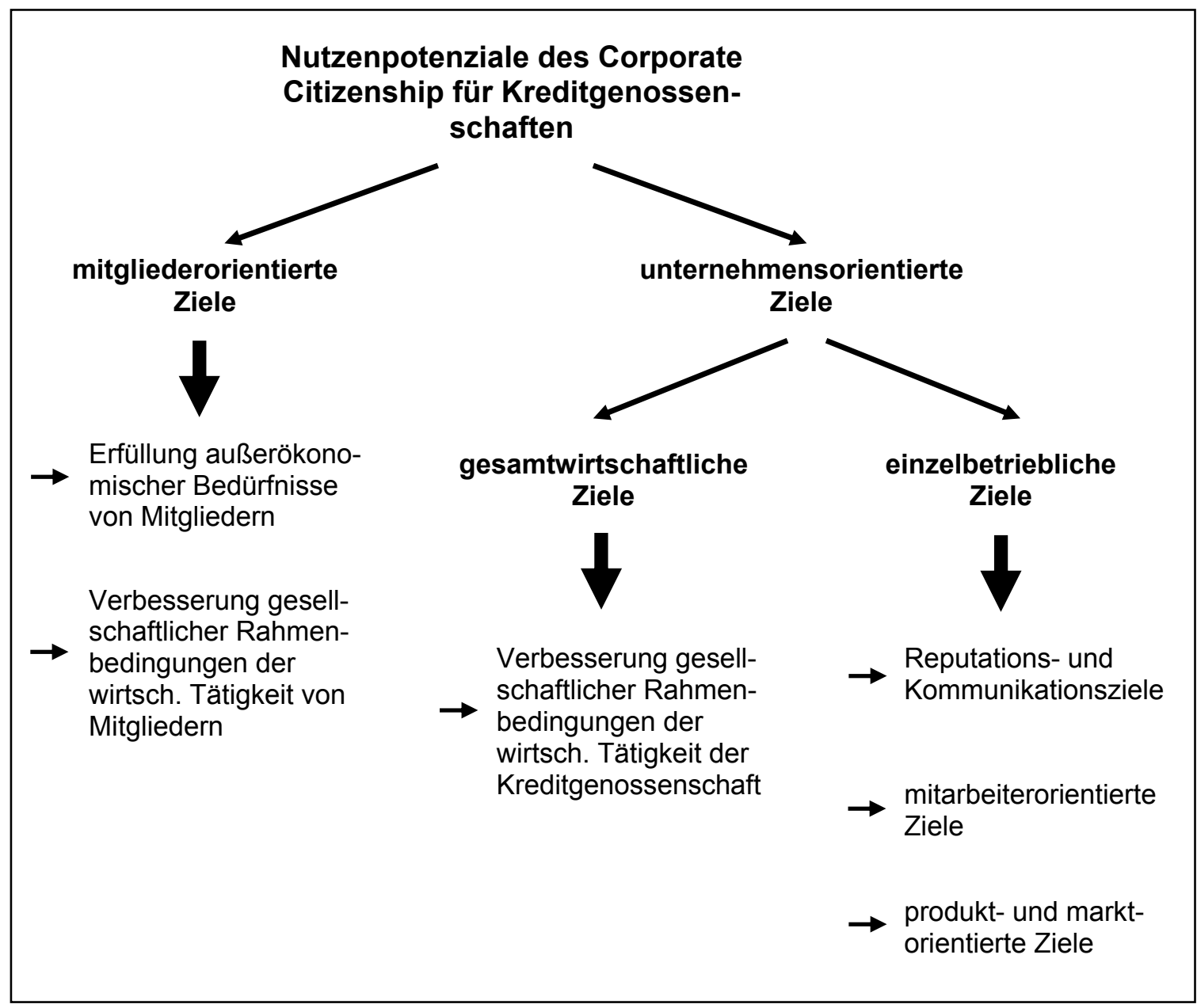

\section{Abb. 1: Nutzenpotenziale des Corporate Citizenship für Kreditgenossenschaften \\ Quelle: $\quad$ Eigene Darstellung}

\section{Literaturverzeichnis}

Aktive Bürgerschaft e.V. (Hrsg.) (2003), Aktive Bürgerschaft - Kompetenz für Bürgerengagement, Berlin

Backhaus-Maul, H. (2004), Corporate Citizenship im deutschen Sozialstaat, in: Aus Politik und Zeitgeschichte, B 14/2004, S. 23-30

Blome-Drees, J. (1998), Strategisches Management als Unternehmungsführungskonzeption von Genossenschaften, Kölner Genossenschaftswissenschaft, Band 24, hrsg. von J. Zerche, Regensburg

Blome-Drees, J. und I. Schmale (2004), Unternehmenskultur von Genossenschaftsbanken - Eine empirische Studie, Neue Kölner Genossenschaftswissenschaft, Band 1, hrsg. von H. J. Rösner und F. Schulz-Nieswandt, Münster

Bonus, H. (1994), Das Selbstverständnis moderner Genossenschaften - Rückbindung von Kreditgenossenschaften an ihre Mitglieder, Tübingen

Bundesministerium für Wirtschaft und Technologie (21. Januar 2006), „Corporate Citizenship - Engagement mittelständischer Unternehmen“, http://www.ccdb-pro-mittelstand.org

Carroll, A. B. (1998), The Four Faces of Corporate Citizenship, in: Business and Society Review 100/101, S. 1-7

Dülfer, E. (1980), Zielsystem der Genossenschaft, in: Handwörterbuch des Genossenschaftswesen, hrsg. von E. Mändle und H. W. Winter, Wiesbaden, Sp. 1857-1872

48 Die Verwendung der Begriffe der erwerbs-, förderungs- und gemeinwirtschaftlichen Orientierung erfolgt hier unter Rekurs auf Engelhardts Widmungstypologie für Genossenschaften (vgl. Engelhardt (1983)). 
Engelhardt, W. W. (1983), Gemeinwirtschaftliche Genossenschaften - ein möglicher Widmungstyp von Genossenschaften unter sechsen, in: Zeitschrift für öffentliche und gemeinwirtschaftliche Unternehmen Bd. 6, Heft 1, S. $30-47$

Englert, D. (2001), Political Dimensions of (Post)Modern Societies, in: Corporate Citizenship as Investing in Social Capital, hrsg. von A. Habisch, H.-P. Meister und R. Schmidpeter, Berlin, S. 33-43

Enquete-Kommission „Zukunft des Bürgerschaftlichen Engagements“ Deutscher Bundestag (Hrsg.) (2002), Bericht - Bürgerschaftliches Engagement auf dem Weg in eine zukunftsfähige Bürgergesellschaft, Opladen

Fabisch, N. (2004), Soziales Engagement von Banken - Entwicklung eines adaptiven und innovativen Konzeptansatzes im Sinne des Corporate Citizenship von Banken in Deutschland, München und Mering

Freeman, R. E. (2004), The Stakeholder Approach Revisited, in: Zeitschrift für Wirtschafts- und Unternehmensethik 5, Heft 3, S. 228-241

Freeman, R. E. (1984), Strategic Management: A Stakeholder Approach, Boston u.a.

Habisch, A. (2003), Corporate Citizenship - Gesellschaftliches Engagement von Unternehmen in Deutschland, Berlin u.a.

Habisch, A. (1998), Social Capital Investments, Property Rights and the Ethics of Win-Win - Why Multinational Enterprise Management Should Engage in Institution Building of their Host Countries, in: Ethics in International Management, hrsg. von B. N. Kumar und H. Steinmann, Berlin und New York, S. 109-126

Habisch, A. und R. Schmidpeter (2001), Social Capital, Corporate Citizenship and Constitutional Dialogues - Theoretical Considerations for Organisational Strategy, in: Corporate Citizenship as Investing in Social Capital, hrsg. von A. Habisch, H.-P. Meister und R. Schmidpeter, Berlin, S. 11-18

Hansen, U., M. Bode und D. Moosmayer (2004), Stakeholder Theory between general and contextual approaches A German view, in: Zeitschrift für Wirtschafts- und Unternehmensethik 5, Heft 3, S. 242-254

Hansen, U. und U. Schrader (2005), Corporate Social Responsibility als aktuelles Thema der Betriebswirtschaftslehre, in: Die Betriebswirtschaft 65, Heft 4, S. 373-395

Haug, S. (1997), Soziales Kapital - Ein kritischer Überblick über den aktuellen Forschungsstand, Arbeitspapiere des Mannheimer Zentrums für Europäische Sozialforschung (MZES), Arbeitsbereich II/Nr. 15, Mannheim

Lang, R. und P. Kromminga (2003), Praktische Erfahrungen mit der Förderung des Engagements von Unternehmen im Gemeinwesen, in: Bürgergesellschaft und Wirtschaft - zur neuen Rolle von Unternehmen, hrsg. v. H. Backhaus-Maul und H. Brühl, Berlin, S. 85-100

Leupold, W. (1975), Die Planung der Formalzielkonzeption in Universalbanken, Köln

Logan, D., D. Roy und L. Regelbrugge (1997), Global Corporate Citizenship - Rationale and Strategies, Washington D.C.

Maßß, F. und R. Clemens (2002), Corporate Citizenship - Das Unternehmen als ,guter Bürger“, Wiesbaden

Mändle, E. (1992), Gemeinwirtschaft und Genossenschaft, Abgrenzung, in: GenossenschaftsLexikon, hrsg. von E. Mändle und W. Swoboda, Wiesbaden, S. 239-240

McIntosh, M. u.a. (1998), Corporate Citizenship - Successful Strategies for Responsible Companies, London u.a.

Näsi, J. (1995), What is stakeholder thinking? A snapshot of a social theory of the firm, in: Understanding Stakeholder Thinking, hrsg. von J. Näsi, Helsinki, S. 17-32

Pauli, H. (1980), Mitgliederstruktur, in: Handwörterbuch des Genossenschaftswesens, hrsg. von E. Mändle und H.-W. Winter, Wiesbaden, Sp. 1202-1215

Pinkepank, T. (2001), „Citizen Can“ - Building Shareholder-Value as a Corporate Citizen - Examples from Germany Showing How Investing in Social Capital Leads to Win-Win Situations, in: Corporate Citizenship as Investing in Social Capital, hrsg. von A. Habisch, H.-P. Meister und R. Schmidpeter, Berlin, S. 114-121

Renner, A. (2001), Social Capital, Corporate Citizenship and the Social Market Economy - The Third Way Politics in Great Britain and in Germany, in: Corporate Citizenship as Investing in Social Capital, hrsg. von A. Habisch, H.-P. Meister und R. Schmidpeter, Berlin, S. 64-75

Richter, D. (1981), Möglichkeiten der Operationalisierung des genossenschaftlichen Förderauftrages - Zur Frage der Ziele, Maßstäbe und Erfolge genossenschaftlicher Arbeit, Düsseldorf

Ringle, G. (1990), Gründe für den Eintritt in eine Genossenschaft, in: Genossenschaftswesen - Hand- und Lehrbuch, hrsg. von J. Laurinkari, München und Wien, S. 452-459

Ringlstetter, M. und M. Schuster (2003), Corporate Citizenship - Eine aktuelle Mode der Strategischen Unternehmensführung, in: Perspektiven der Strategischen Unternehmensführung: Theorien - Konzepte - Anwendungen, hrsg. von M. Ringlstetter, H. A. Henzler und M. Mirow, Wiesbaden, S. 169-198

Rösner, H. J. (1997), Gibt es eine genossenschaftliche Unternehmenskultur? in: Zeitschrift für das gesamte Genossenschaftswesen 47 , S. 260-272

Schmidpeter, R. (2001), InnoRegio Networks as Social Capital of the Region, in: Corporate Citizenship as Investing in Social Capital, hrsg. von A. Habisch, H.-P. Meister und R. Schmidpeter, Berlin, S. 122-127

Schrader, U. (2003), Corporate Citizenship - Die Unternehmung als guter Bürger?, Berlin

Schwarz, P. (2005), Genossenschaftsmanagement im Dilemma zwischen Profit-, Nonprofit- und Gemeinwohlorientierung, in: Das Genossenschaftswesen in der Schweiz, hrsg. von P. Purtschert, Bern u.a., S. 193-217

Thiemeyer, T. (1990), Zur Abgrenzung von Genossenschaften und Gemeinwirtschaft, in: Genossenschaftswesen Hand- und Lehrbuch, hrsg. von J. Laurinkari, München und Wien, S. 324-336 
Ulrich, P. (2002), Republikanischer Liberalismus und Corporate Citizenship - Von der ökonomistischen Gemeinwohlfiktion zur republikanisch-ethischen Selbstbindung wirtschaftlicher Akteure, Bericht des Institut für Wirtschaftsethik Nr. 88, St. Gallen

Wagner, A. (1999), Unternehmensethik in Banken, Wien

Weisser, G. (1977), Öffentliche Aufgaben von Genossenschaften, in: Zeitschrift für das gesamte Genossenschaftswesen 27, S. 401-413

Westebbe, A. und D. Logan (1995), Corporate Citizenship - Unternehmen im gesellschaftlichen Dialog, Wiesbaden Wieland, J. (2002), Corporate Citizenship-Management - Eine Zukunftsaufgabe für die Unternehmen!? in: Corporate Citizenship: Gesellschaftliches Engagement - unternehmerischer Nutzen, hrsg. von J. Wieland und W. Conradi, Marburg, S. 9-21 